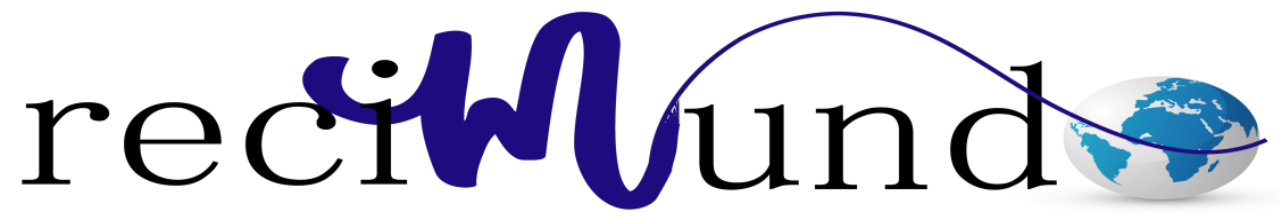

Revista Científica Mundo de la Investigación y el Conocimiento

Milton Israel Manosalvas Tola ${ }^{\text {a; }}$ Luis Alemán Herrera Lozano ${ }^{\text {; }}$; Segundo Olimpo Cárdenas Romero ${ }^{\mathrm{c}}$

El Estudio de caso como metodología para el desarrollo de la Inteligencia Hermeneutica-Pragmatica en Ciencias Administrativas

Revista Científica Mundo de la Investigación y el Conocimiento. Vol. 2 núm., 1, febrero, ISSN: 2588-073X, 2018, pp. 362-373

DOI: $\underline{10.26820 / \text { recimundo/2.1.2018.362-373 }}$

Editorial Saberes del Conocimiento

Recibido: 05/12/2017

Aceptado: 10/02/2018

a. Ingeniero Comercial, Magister en Administración de Empresas, Docente Universidad de Guayaquil; milton.manosalvasto@ug.edu.ec

b. Docente Universidad de Guayaquil; luis.herreral@ug.edu.ec

c. Docente Universidad de Guayaquil. 


\section{El Estudio de caso como metodología para el desarrollo de la Inteligencia Hermeneutica-Pragmatica en Ciencias Administrativas}

Vol. 2, núm. 1., (2018)

Milton Israel Manosalvas Tola; Luis Alemán Herrera Lozano; Segundo Olimpo Cárdenas

Romero

\section{RESUMEN}

El propósito de este artículo es establecer pautas, métodos, estructuras para la utilización de un instrumento pedagógico como el Estudio de Casos para el desarrollo de la inteligencia hermenéutica- pragmática para su aplicación en la administración estratégica y o en la Ciencias sociales. El valor de esta herramienta se basa en afianzar los conocimientos teóricos adquiridos con una situación problemática de una organización en particular, a través de encontrar soluciones que podrían inclusive servirles a empresas con características similares. Para este trabajo se utilizó el estudio de caso como metodología pedagógica a una comunidad de estudiantes y se demostró en forma cualitativa, cuantitativa que esta no solo afianza los conocimientos teóricos adquiridos sino que desarrolla el espíritu grupal reflexivo e investigativo. Además de proponer una matriz que permita a los administradores en una forma simplificada hacer un diagnóstico del problema y toma las decisiones conforme a los valores compartidos y los objetivos de la empresa.

Palabras clave: Administración, Inteligencia, Hermenéutica, Pedagogía, Organización. 


\section{El Estudio de caso como metodología para el desarrollo de la Inteligencia Hermeneutica-Pragmatica en Ciencias Administrativas}

Vol. 2, núm. 1., (2018)

Milton Israel Manosalvas Tola; Luis Alemán Herrera Lozano; Segundo Olimpo Cárdenas Romero

\section{ABSTRACT}

The purpose of this article is to establish guidelines, methods, structures for the use of a pedagogical instrument such as Case Studies for the development of hermeneutic-pragmatic intelligence for its application in strategic management and in social sciences. The value of this tool is based on consolidating the theoretical knowledge acquired with a problematic situation of a particular organization, through finding solutions that could even serve companies with similar characteristics. For this work the case study was used as a pedagogical methodology for a community of students and it was demonstrated in a qualitative, quantitative way that this not only strengthens the acquired theoretical knowledge but also develops the reflective and investigative group spirit. In addition to proposing a matrix that allows managers in a simplified way to diagnose the problem and make decisions according to the shared values and objectives of the company.

Keywords: Administration, Intelligence, Hermeneutics, Pedagogy, Organization. 


\section{El Estudio de caso como metodología para el desarrollo de la Inteligencia Hermeneutica-Pragmatica en Ciencias Administrativas}

Vol. 2, núm. 1., (2018)

Milton Israel Manosalvas Tola; Luis Alemán Herrera Lozano; Segundo Olimpo Cárdenas

Romero

\section{Introducción.}

En las actividades que el hombre realiza, se deben tomar decisiones especialmente cuando el individuo se encuentra en una variación que altera la situación ideal, estas decisiones se toman en forma planificada de acuerdo a la información que se posee, aunque la mayoría de la veces ellas se las realiza inconscientemente o conscientemente de acuerdo a la perspectiva del riesgo permitido.

En la toma de decisiones el administrador lo hace de acuerdo a diferentes niveles del conocimiento que se combinan, El óntico, que se refiere al raciocinio a la calidad de interpretación de acuerdo a nivel ontológica en la que emplea los medios de información, que combinados forman el desarrollo hermenéutico.

La administración de las organizaciones están directamente ligadas al desarrollo de la sociedad, es así que en el primer estado la administración se refería a cuestiones prácticas y utilitarias .Los primeros estudios de Administración se refirieron a estudios prácticos que buscaban aumentar la productividad de los trabajadores, los mismos que fueron desarrollados por Taylor. En 1916 Henry Fayol en su obra Principio Generales de Administración plantea la administración como un cuerpo social en la cual existen reglas y principios. Peter Drucker establece un método o sistemas para tomar decisiones basadas en el FODA, en la que plantea el estudio del ambiente externo e interno de la organización.

La diferente interpretación filológica, teológica, jurídica de los libros surge desde tiempos remotos, pero fue (Gadamer, 1986) fundador de la escuela hermenéutica, que indica que siempre 


\section{El Estudio de caso como metodología para el desarrollo de la Inteligencia Hermeneutica-Pragmatica en Ciencias Administrativas}

Vol. 2, núm. 1., (2018)

Milton Israel Manosalvas Tola; Luis Alemán Herrera Lozano; Segundo Olimpo Cárdenas Romero

que nos acercamos a un texto, lo hacemos a partir de un proyecto, con alguna idea previa de lo que allí se dice. A medida que estudiamos, este proyecto va variando y se va reformulando según la lectura nos vaya confirmando o alterando nuestra pre- comprensión. Como este proceso puede prolongarse al infinito, nunca podemos afirmar que hemos dado la interpretación última y definitiva. (Morella, Calles, \& Moreno de Tovar, 2006)

En el momento de la toma de decisiones de acuerdo a (Peirce, 1904) "la acción es un principio de acceso a la realidad" y que cualquier acción de un sujeto a un objeto, llamase entorno produce una reacción que genera nuevas situaciones y que en las organizaciones la tarea del administrador al tomar acciones produce nuevas reacciones que se incorporan generando nuevos conocimientos, a este proceso de conocimiento se lo conoce como pragmatismo.

Ante la complejidad de la toma de decisiones el administrador debe desarrollar a través de la inteligencia hermenéutica - pragmática un conjunto de repuestas

En la toma de decisiones el administrador se encuentra en una sociedad de cambios dinámicos, por tanto las decisiones, están sujetas a la dialéctica del conocimiento, por tanto no bastan los antiguos métodos de investigación, como el deductivo, inductivo o el brain storming en administración. Es por eso que el estudio teórico de la administración, debe ser complementados con casos prácticos que simulen las situaciones reales que el estudiante enfrentara en el mundo real y moderno, ya que su toma de decisiones frecuentemente afectaran no solo las organizaciones sino todo el contorno y el sistema que se desenvuelven desde el aspecto económico, social y los valores culturales. 


\section{El Estudio de caso como metodología para el desarrollo de la Inteligencia Hermeneutica-Pragmatica en Ciencias Administrativas}

Vol. 2, núm. 1., (2018)

Milton Israel Manosalvas Tola; Luis Alemán Herrera Lozano; Segundo Olimpo Cárdenas

Romero

El estudio de caso por medio de la matriz metodológica de resolución indica la forma como, cuando y porque debemos cambiar la estrategia al encontrarnos en una situación afectiva, proponiendo soluciones conforme a los valores corporativos.

De gran aporte para la construcción de la matriz de resolución de casos es el Cuadro de Mando, llamado también The Balance Scorecard de (Norton, 2010) "en la que describe el mapa estratégico del cuadro de mando integral para determinar e identificar los indicadores de gestión que la empresa tiene. En base a ello se puede determinar los Indicadores Claves del Éxito.

En el estudio de casos se deben plantear preguntas científicas sobre el Porqué, Como, Para, que justifican la investigación y que están contempladas en el Tema del Estudio de Caso.

El método de estudio de caso es una forma de hacer investigaciones basadas, en observaciones, encuestas, historia, conductas sociales, épicas. Las limitaciones o desventajas, son las experimentales por las implicaciones éticas o por que los resultados no corresponderían a una realidad social económica.

Para el presente trabajo se define el Estudio de Caso, Como un método de investigación para formulación de procesos administrativos y reducir las brechas o conflictos entre lo actual y lo deseado en un desarrollo sistemático de interacción del aprendizaje de las ciencias administrativas.

Tipos de Casos según los objetivos que se persigue. 


\section{El Estudio de caso como metodología para el desarrollo de la Inteligencia Hermeneutica-Pragmatica en Ciencias Administrativas}

Vol. 2, núm. 1., (2018)

Milton Israel Manosalvas Tola; Luis Alemán Herrera Lozano; Segundo Olimpo Cárdenas Romero

\section{Explicativos.}

Cuando necesitamos reforzar los conocimientos teóricos escogemos un caso de una empresa que ha implementado un programa o una solución en una situación determinada, no importa si esta haya sido de éxito o fracaso, se puede plantear preguntas.

- Cuáles fueron las causas de la solución planteada

- Que situaciones no se validaron

- Como reaccionaron los grupos interesados.

\section{Descriptiva}

En este el estudio de caso describe lo que está sucediendo con el propósito de obtener información sobre los diferentes indicadores y circunstancias que rodea al área critica del problema, sirve para comprender las interacciones de las variables que afectan el proceso administrativo, con el objeto de formular teorías administrativas.

\section{Aportaciones Administrativas}

Este estudio de caso estudia todas las variables, indicadores, estructuras, modelos administrativos que hay sido diseñados para comprender y diagnosticar los procesos administrativos. FODA, PEST, para realizar un diagnóstico y hacer una propuesta de solución al 


\section{El Estudio de caso como metodología para el desarrollo de la Inteligencia Hermeneutica-Pragmatica en Ciencias Administrativas}

Vol. 2, núm. 1., (2018)

Milton Israel Manosalvas Tola; Luis Alemán Herrera Lozano; Segundo Olimpo Cárdenas

Romero

problema, con este modelo se aporta con nuevas herramientas empíricas que se podrían contrastar con la práctica para convertirse en teorías administrativas

A partir del referido concepto se planteó la metodología de estudio de casos de administración para los estudiantes de la Facultad de Ciencias Administrativas de la Universidad de Guayaquil, cuyos objetivos fueron

- Reforzar los conocimientos teóricos con situaciones complejas de prácticas en empresas

- Simular situaciones de empresas con problemas o iniciativas de proyectos emprendedores, para las construcciones de estos estudios se requiere de un conocimiento teórico y experiencia práctica profesional del docente instructor teniendo presente el objetivo que se quiere evaluar.

- Fomentar el espíritu crítico reactivo entre los participantes.

- El estudiante se acerca a la realidad del futuro entorno empresarial como tomador de decisiones en enfoques problemáticos

- Generación de conocimientos a través del desarrollo de la inteligencia hermenéutica -pragmática, ya que todo conocimiento es válido cuando se lo vincula con acción práctica.

Se debe indicar que este modelo de aprendizaje supero las expectativas planteadas de acuerdo a los criterios emitidos por los participantes ya que se consiguió un altísimo nivel de 


\section{El Estudio de caso como metodología para el desarrollo de la Inteligencia Hermeneutica-Pragmatica en Ciencias Administrativas}

Vol. 2, núm. 1., (2018)

Milton Israel Manosalvas Tola; Luis Alemán Herrera Lozano; Segundo Olimpo Cárdenas Romero

cooperación y sirvió de estímulo para la investigación y revisiones del estudio del arte en la Administración y de otras disciplinas adyacentes.

\section{Modelo de Estructura para el Estudio de Caso.}

Aunque los casos no están sujetos a una estructura rígida con el fin de que el proceso sea más metódico se propuso una estructura que recoge los principales lineamientos de las empresas y una matriz de resolución de casos que responde a las preguntas de la estrategia competitiva de acuerdo a (Thompson, 2015) “¿Cuál es nuestra situación actual? ¿Hacia dónde queremos ir? ¿Cómo vamos a llegar?” La matriz de resolución de casos plantea responder a esas preguntas con un solo bosquejo. Todo ello tomando como referencia el Lienzo de Negocios del Modelo lo de Negocios CANVAS de (Osterwalder A., 2015)

\section{Materiales y métodos.}

Para este trabajo se utilizó el estudio de caso como metodología pedagógica a una comunidad de estudiantes. En el periodo 2015 al 2016 se procedió a utilizar la metodología del estudio de casos a trecientos estudiantes de la Facultad de Ciencias Administrativas de la Universidad de Guayaquil por medio la aplicación de evaluaciones y encuestas.

\section{Resultados.}

- Los estudiantes en un 67\%, al inicio del curso indicaron que sus conocimientos en ciencias administrativas eran buenos.

- Los estudiantes en un79\%, manifestaron que es excelente la estimulación para el estudio el uso de esta herramienta, el 11\% indico que era muy buena y el $10 \%$ regular. 


\section{El Estudio de caso como metodología para el desarrollo de la Inteligencia Hermeneutica-Pragmatica en Ciencias Administrativas}

Vol. 2, núm. 1., (2018)

Milton Israel Manosalvas Tola; Luis Alemán Herrera Lozano; Segundo Olimpo Cárdenas

Romero

- El $80 \%$ de los estudiantes respondieron que esta metodología del estudio de caso los ayudo a comprender los procesos administrativos en la solución de los problemas.

- El $67 \%$ de los estudiantes encontraron que sus relaciones interpersonales fueron excelentes con los estudios de casos, el $17 \%$ contesto muy bien y el $16 \%$ bien.

- El estudio de caso como herramienta para evaluar los conocimientos en administración los estudiantes el 50\% lo consideran excelente, el 33\% muy bueno, el 13\% bueno y un $3.3 \%$ regular.

- El $47 \%$ de los entrevistados califico como excelente para reforzar los estudios y el $40 \%$ lo califico de muy bueno, el $10 \%$ bueno y el 3,3\% regular.

- El $67 \%$ de los entrevistados manifestaron que la Metodología del estudio de caso y su estructura los motivo a estudiar todo lo relacionado con las Ciencias Administrativas.

- El $87 \%$ de los entrevistados manifestaron que la Metodología de estudios de casos lo considera excelente para el trabajo de grupos en clases.

\section{Conclusiones.}

- De acuerdo a la encuesta realizada a los estudiantes y al proceso enseñanza aprendizaje en las aulas se puede concluir que la metodología del estudio de caso en Ciencias Administrativas, permite que los estudiantes al aplicar sus conocimientos teóricos a un caso específico, los prepara para el campo profesional, además que es una fuente de estimulación para la investigación, debido a que tienen que indagar en algunas áreas de la ciencias administrativas. 


\section{El Estudio de caso como metodología para el desarrollo de la Inteligencia Hermeneutica-Pragmatica en Ciencias Administrativas}

Vol. 2, núm. 1., (2018)

Milton Israel Manosalvas Tola; Luis Alemán Herrera Lozano; Segundo Olimpo Cárdenas Romero

- La matriz para la resolución de casos permite de un solo bosquejo comprender el proceso administrativo de la empresa, sus fortalezas, debilidades, procesos de producción, para determinar las brechas que existen en las áreas críticas y recomendar las alternativas de solución, involucrando a las áreas perspectivas. Es una herramienta que no solo consiste en conocer los resultados de las organizaciones sino que induce a presentar una estrategia de acuerdo a los recursos, valores compartidos, objetivos empresariales y grupos interesados.

- La Matriz de resolución de casos es una recopilación de la gestión administrativa de la empresa en un diagrama que permite en forma estructural conocer toda la gestión administrativa de la empresa, sus principales indicadores, cómo los recursos, contribuyen a los objetivos de la empresa, las holguras o brechas del sistema empresarial del sistema empresarial. En base a ello se puede determinar las alternativas de solución y la solución recomendada, así como los controles y evaluaciones que es necesario implementar.

\section{Recomendaciones.}

- Es de destacar que el estudio de caso de empresas se puede simular un caso de acuerdo al objetivo que se desea medir, para ello el coaching debe tener mucha experiencia práctica profesional y académica. 


\section{EI Estudio de caso como metodología para el desarrollo de la Inteligencia Hermeneutica-Pragmatica en Ciencias Administrativas}

Vol. 2, núm. 1., (2018)

Milton Israel Manosalvas Tola; Luis Alemán Herrera Lozano; Segundo Olimpo Cárdenas

Romero

- Se debería continuar con esta metodología de estudio de casos, especialmente en los años de estudios superiores de las carreras de ciencias sociales, de la administración, para preparar al estudiante con la praxis profesional.

\section{Bibliografía.}

Gadamer, H. G. (1986). VERDAD Y MÉTODO: FUNDAMENTOS DE UNA HERMENÉUTICA FILOSÓFICA. Nueva York: Wahrheit und Methode.

Morella, A., Calles, J., \& Moreno de Tovar, L. (2006). La Hermenéutica:una actividad interpretativa. Sapiens. Revista Universitaria de Investigación, 7(2), 171-181.

Norton, R. S. (2010). Cuadro de Mando Integral. En R. S. Norton, Cuadro de Mando Integra.

Osterwalder, A. (2015). Generacion de Modelos de Negocios. México: Grupo Planeta Spain.

Thompson, A. A. (2015). Administracion Estrategica. Alabama: Mc. Graw Hill. 